\title{
Successful thoracoscopic management of iatrogenic left subclavian arterial injury: a case report
}

\author{
Yunke Zhu ${ }^{1,2 \#}$, Quan Zheng ${ }^{1,2 \#}$, Hu Liao ${ }^{1,2}$, Jiandong Mei ${ }^{1,2}$, Gregor J. Kocher ${ }^{3}$, Kimihiro Shimizu ${ }^{4}$, Lin $^{\text {Ma }}{ }^{1,2}$ \\ ${ }^{1}$ Department of Thoracic Surgery, West China Hospital, Sichuan University, Chengdu, China; ${ }^{2}$ Western China Collaborative Innovation Center \\ for Early Diagnosis and Multidisciplinary Therapy of Lung Cancer, Sichuan University, Chengdu, China; ${ }^{3}$ Division of General Thoracic Surgery, \\ Inselspital, Bern University Hospital, University of Bern, Bern, Switzerland; ${ }^{4}$ Division of General Thoracic Surgery, Department of Surgery, Shinshu \\ University School of Medicine, Asahi, Matsumoto, Nagano, Japan \\ "These authors contributed equally to this work. \\ Correspondence to: Lin Ma. Department of Thoracic Surgery, West China Hospital, Sichuan University, No. 37, Guoxue Alley, Chengdu 610041, \\ China. Email: malin1982526@163.com.
}

\begin{abstract}
The subclavian artery at the thoracic outlet is in the deepest position of the thoracic cavity and is difficult to repair in this narrow space once injured, even if the surgery is converted to a thoracotomy. This article presents a successful left subclavian artery repair procedure at the thoracic outlet using a thoracoscopic approach, with a video demonstration, and describes its technical characteristics. The patient was planned for a left upper lobectomy through three-port thoracoscopic approach. Severe adhesions were found intraoperatively and an accidental left subclavian arterial injury occurred when dissecting the adhesions. We first clamped the proximal portion of the subclavian artery and then directly clamped the rupture site. Our first suture failed due to the limited suture angle and the mutual restriction between the needle holder and atraumatic vascular clamp. To freely control the needle holder, another assistant port was made in the seventh intercostal space (ICS). The arterial injury was finally successfully repaired using pledgetted suture. The operation time was 235 minutes and intraoperative blood loss was $800 \mathrm{~mL}$. The pulsation of the left radial artery was normal postoperatively, and the patient was discharged on postoperative day 6. Appropriate strategies allow attempts to manage intraoperative hyperbaric arterial bleeding from the systemic circulation, such as bleeding caused by subclavian arterial injuries, by means of a thoracoscopic approach without conversion to thoracotomy.
\end{abstract}

Keywords: Thoracoscopy; subclavian arterial injury; massive hemorrhage; case report

Submitted Apr 24, 2021. Accepted for publication Dec 24, 2021.

doi: $10.21037 /$ jtd-21-1922

View this article at: https://dx.doi.org/10.21037/jtd-21-1922

\section{Introduction}

Massive hemorrhages caused by vascular injury are the most dangerous complications during thoracoscopic lung surgery. The first instinct of thoracoscopic surgeons was conversion to thoracotomy when facing major hemorrhage during surgery. Several effective methods to address bleeding, such as the use of thrombostatic material and biological sealants, or the discreet utilization of energy sources, have been summarized (1). We have described a series of strategies for bleeding pulmonary vessels during thoracoscopic lung surgery (2). However, managing severe bleeding from hyperbaric systemic arteries remains challenging even for experienced thoracoscopic surgeons and has been rarely reported. The subclavian artery at the thoracic outlet is in the deepest position of the thoracic cavity and is difficult to repair in this narrow space once injured, even if the surgery is converted to a thoracotomy. This article presents a successful left subclavian artery repair procedure at the thoracic outlet under the thoracoscopic approach with a video demonstration (Video 1) and describes its technical characteristics. Although the present case was planned for 


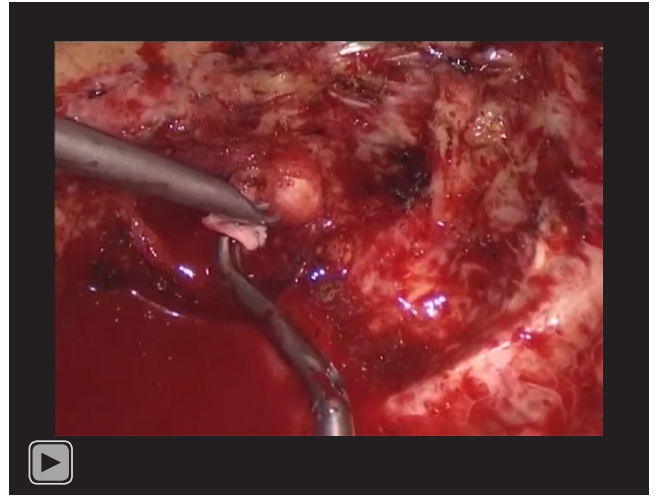

Video 1 Thoracoscopic management of left subclavian arterial injury at the thoracic outlet.

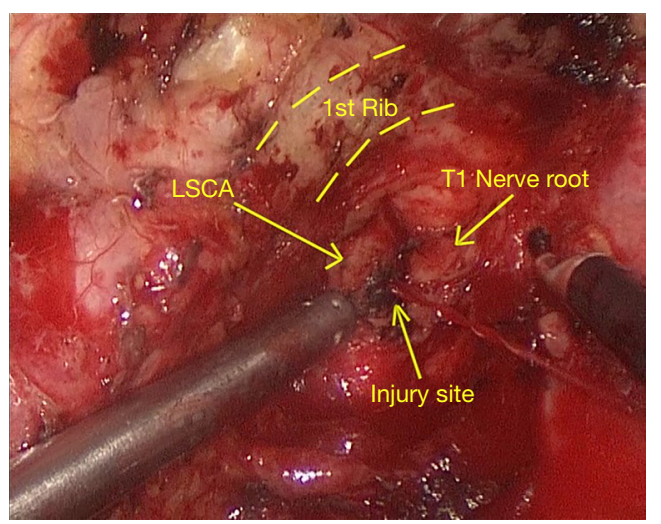

Figure 1 An accidental intraoperative left subclavian arterial injury at the thoracic outlet occurred during the dissection of severe pleural adhesions. LSCA, left subclavian artery.

a three-portal thoracoscopic surgery, the experience we shared here might as well be referenced during two-portal or uniportal thoracoscopic surgery.

We presented the following article in accordance with the CARE reporting checklist (available at https://jtd. amegroups.com/article/view/10.21037/jtd-21-1922/rc).

\section{Case presentation}

A 68-year-old man was admitted to our hospital for a mass in the left upper lobe with high suspicion of lung cancer. The preoperative oncological evaluation of this patient revealed a clinical-stage of cT1N0M0. Left upper lobectomy through a three-portal thoracoscopic approach was planned for this patient, as we previously described (3). However, severe adhesions were found between the left upper lobe and the thoracic outlet. An accidental left subclavian arterial injury at the thoracic outlet occurred when the adhesions were dissected using a hook (Figure 1). Given our experience in managing thoracoscopic vascular injury (2), we decided to try thoracoscopic repair. However, we were also prepared for the thoracotomy simultaneously.

First, we mobilized the proximal portion of the subclavian artery and used a releasable endoscopic bulldog clamp (Aesculap, Inc., Center Valley, PA, USA) to block it for subsequent suture (4). However, there was no space to clamp the distal portion of this artery. We clearly exposed the vascular wound, and suturing remained difficult without controlling the hemorrhage. Thus, we clamped the rupture site directly using an endoscopic atraumatic vascular clamp (Aesculap, Inc., Center Valley, PA, USA) to stop bleeding from the assistant port in the ninth intercostal space (ICS). However, our first suture failed due to the complicated problem of the limited suture angle and the mutual restriction between the needle holder and the atraumatic vascular clamp, both inserted in the same operating port. The bleeding worsened after the first suture. Therefore, another assistant port was made in the seventh ICS to facilitate suturing. We could control the needle holder to complete the suture freely with this added port. Finally, the left subclavian arterial injury was successfully repaired using a pledgetted suture (4/0 Prolene stitch).

After successfully treating the bleeding, a left upper lobectomy was performed with thoracoscopic and the diagnosis of adenocarcinoma was confirmed by frozen section. The total operation time was 235 minutes and the blood loss was $800 \mathrm{~mL}$. The patient's left radial artery pulsation was normal postoperatively. No complications were observed. The chest tube was removed on postoperative day 5 and the patient was discharged on postoperative day 6 (Figure 2).

All procedures performed in this study involving human participants were in accordance with the ethical standards of the institutional research committee and with the Helsinki Declaration (as revised in 2013). Written informed consent was obtained from the patient for publication of this case report and accompanying images. A copy of the written consent is available for review by the 


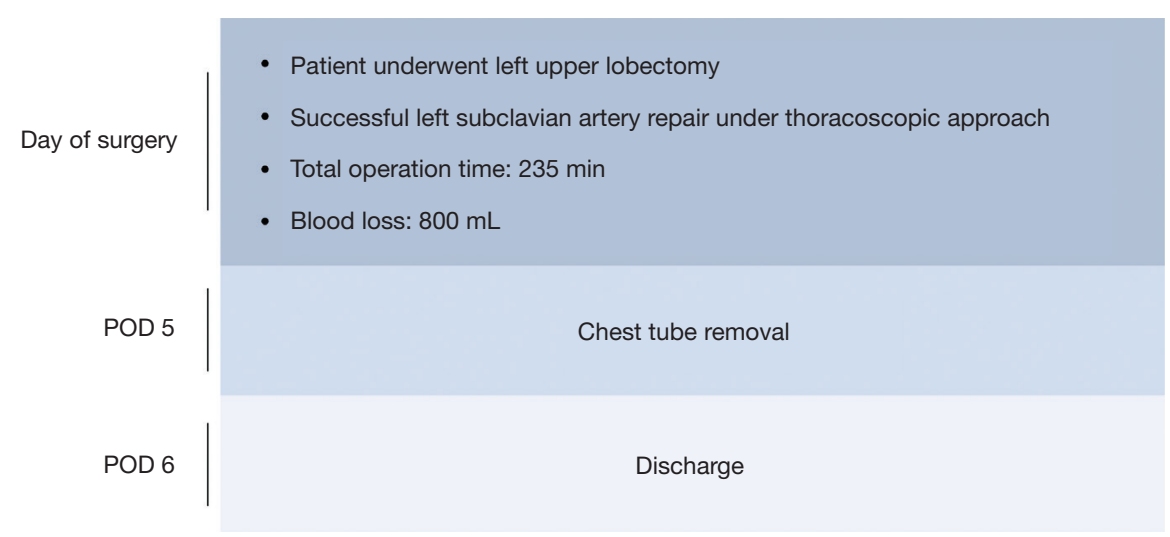

Figure 2 Timeline for hospitalization. POD, postoperative day.

editorial office of this journal.

\section{iMDT discussion}

\section{Discussion among physicians from West China Hospital}

\section{Department of Thoracic Surgery}

In most cases, bleeding from iatrogenic subclavian artery injuries, especially lacerations located at the thoracic outlet, is catastrophic. Tam et al. reported the first successful thoracoscopic suture of a subclavian artery injury caused by central venous catheterization (5), but did not provide sufficient technical details.

In this case, we made a bold but not reckless attempt. Our video revealed that an appropriate strategy could be applied to address hyperbaric arterial injury at the thoracic outlet during thoracoscopic surgery. Our experience showed that the thoracoscopic treatment of large-vesselinjured hemorrhage has the following key points: first, when a vascular injury occurs, the surgeons must remain calm and avoid clamping the bleeding vessels blindly. After controlling bleeding by direct compression, some lifesaving interventions must be prepared, such as alerting the anesthesia team to have blood products in the room and building large-bore intravenous access. Second, surgeons must adopt effective techniques, such as blocking vessels or clamping the injury site, and controlling bleeding to provide a visual field for subsequent sutures. In particular, clamping an injured vessel with an atraumatic vascular clamp must be done with considerable caution, and locking the ratchet too tightly should be avoided, because this may cause additional injury to the vascular wall. Third, the addition of an assistant port may provide more space and possibly a better angle for thoracoscopic bleeding control, especially in narrow areas such as the thoracic outlet.

The major limitation for thoracoscopic management of large-vessel-injured hemorrhage is its high demand for surgical skills. The approach depended very much on the experience of the surgeon plus the whole medical team. Thus the surgeons should consider their surgical skills in managing great vessels when using a thoracoscopic approach. Conversion to open surgery should always be acceptable and prepared.

\section{Several issues regarding the diagnosis and treatment of this patient were further discussed as follows}

\section{Question 1: What type of life-saving interventions should be prepared when a large vessel injury happens intraoperatively?}

Expert opinion 1: Dr Gregor 7. Kocher

The first step when being confronted with substantial intraoperative bleeding is always bleeding control. The most effective way to do this is to compress the bleeding using a peanut or a larger gauze ball for compression. Accordingly, such instruments should always be ready at the operating room (OR) table to be used within seconds. Once the bleeding is temporarily under control the situation can be assessed in a calm manner, discussing the options. These should include the following: is there a senior specialist surgeon available that has more experience and can help resolve the matter? If not, is there another senior surgeon available that can at least assist in the surgery to help, in terms of 'another set of experienced hands in the OR'?

For any surgery with the risk for bleeding complications, these questions should already be addressed before 
surgery-who do I call when I get into trouble during surgery?

If for example vascular 'injury' seems to be very likely due to possible tumor infiltration, the case should also be discussed with a vascular surgeon in order to properly plan the procedure. For example in case of suspected tumor invasion of large vessels such as aorta or subclavian vessels, intraoperative - or in some cases even preoperative endovascular stent placement may be a valid option. In some cases also en bloc resection with vascular reconstruction might be necessary-again, the presumably needed materials should be available [i.e., polytetrafluoroethylene (PTFE) vascular prosthesis and/or pericardial patch].

Depending of the situation, preoperative guide wire placement into the femoral artery and/or vein may facilitate and allow for a timely endovascular stent implantation.

For bleeding control the appropriate instruments should be within reach in the OR: vascular clamps (including bulldog devices), endoscopic needle holder for suturing, rib spreader in case of emergency thoracotomy, bleeding control agents such as Tachosil ${ }^{\circledR}$, Veriset ${ }^{\mathrm{TM}}$ etc.

\section{Expert opinion 2: Dr. Kimibiro Sbimizu}

The most important thing is to be prepared for emergency blood transfusion, to gather medical staff, and to have a cardiovascular surgeon on call. However, in cases when large vessels, including pulmonary artery, are injured, percutaneous cardiopulmonary support (PCPS) would be eventually necessary. Therefore, in our institute, we have a system in place that allows the use of PCPS during any surgery, so that we can tackle the worst-case scenario. We also place patients in the lateral position with the lower part of the body twisted slightly upwards, so that a PCPS catheter can be immediately inserted when necessary. Moreover, we train every six months (including surgeons, anesthesiologists, surgical nurses, and medical engineers) and we simulate clinical cases which require emergency intraoperative PCPS, so that each staff gets sufficiently prepared for such cases.

Question 2: In a situation like this case, should we choose to continue thoracoscopically handling? Or does conversion to open thoracotomy have advantages over thoracoscopic management?

\section{Expert opinion 1: Dr Gregor 7. Kocher}

As discussed by the authors, this decision is very much dependent on the experience of the surgeon, and or other (senior) surgeons of the team. Sometimes conversion to open thoracotomy does not really improve the exposure of the bleeding site - on the contrary, precious time might be lost for creating a thoracotomy, when actually the bleeding could have been stopped more easily using endoscopic measures.

\section{Expert opinion 2: Dr. Kimibiro Shimizu}

It would be basically safer to convert to open thoracotomy in such situation. However, clamping the left subclavian artery could be relatively safer when compared to right brachiocephalic artery because it does not affect cerebral circulation. From that point of view, the present case could be an educational case for future reference. On the other hand, it should be noted that if the area of rupture gets wider during procedure, conversion to thoracotomy is urgently required.

\section{Question 3: Compared with arteries in pulmonary} circulation, arteries in systemic circulation had higher pressure, and their accident rupture would be more lethally. What are the optimal managements thoracoscopically for artery rupture of systemic circulation? or do we have more experiences? Expert opinion 1: Dr Gregor F. Kocher

While the pulmonary artery system is a low pressure system and small bleedings can even be controlled by simple compression for a minute or two, the vessel walls are very thin and injury may happen even during low force manipulation with a blunt instrument.

On the other hand, systemic arterial injuries usually do not stop with compression only, but more force is needed to cause a relevant bleeding.

In both scenarios (for pulmonary as well as for systemic arterial injury), suturing of the bleeding site is a good and valid option. However, sometimes the bleeding site is not easily accessible-as in the reported case. In case of middle sized lesions of the pulmonary artery, sealants such as Tachosil ${ }^{\circledR}$ and Veriset $^{\mathrm{TM}}$ have proven to be effective, easily applicable and safe. In case of systemic arterial injury the burst pressures are much higher, therefore suturing or a thicker material such as Veriset $^{\mathrm{TM}}$ needs to be used. This would have also been a very valid option for the presented case.

\section{Expert opinion 2: Dr. Kimibiro Shimizu}

We have previously experienced a case of intraoperative bleeding from the right brachiocephalic artery. The bleeding occurred while adhesiolysis at the right thoracic outlet. We initially did not know from which part the bleeding occurred, so we could not judge where to suture. However, the wound gradually got larger and led to massive bleeding, which enabled us to realize that the bleeding 
occurred from the right brachiocephalic artery. After we failed to achieve hemostasis with compression using cotton gauze and $\mathrm{TachoSil}^{\circledR}$, we converted to thoracotomy and manually compressed the bleeding site. We first dissected the adhesion of the right upper mediastinum and performed upper mediastinal dissection procedure, as for a usual lymph node dissection for lung cancer, in order to remove lymph nodes and fatty tissue of the mediastinum. We then exposed and clamped the brachiocephalic artery in order to suppress blood flow. We then closed the rupture site by direct suture. We believe that surgical suture under thoracotomy is a safer choice in a critical situation, especially when bleeding from the right brachiocephalic artery. Total-clamp of brachiocephalic artery necessitates careful handling because it may cause decrease in cerebral blood flow. Although clamping the proximal part may decrease the amount of bleeding, there will still be significant bleeding during suture because there is usually a connection between right and left cerebral vessels, which induces backflow. Based on this experience, bleeding from the right brachiocephalic artery is an indication for emergent thoracotomy at our institute.

\section{Conclusions}

Thoracoscopic approach is feasible to manage intraoperative large-vessel-injured hemorrhage with appropriate strategies. Although it depended on surgeon's experience, thoracoscopic approach could save precious time while achieve comparable exposure of bleeding site compared with conversion to thoracotomy.

\section{Acknowledgments}

Funding: This study was supported by the Basic Science Program (No. 2018JY0596 to Lin Ma), Science \& Technology Department of Sichuan Province.

\section{Footnote}

Reporting Checklist: The authors have completed the CARE reporting checklist. Available at https://jtd.amegroups.com/ article/view/10.21037/jtd-21-1922/rc

Conflicts of Interest: All authors have completed the ICMJE uniform disclosure form (available at https://jtd.amegroups. com/article/view/10.21037/jtd-21-1922/coif). The authors have no conflicts of interest to declare.
Ethical Statement: The authors are accountable for all aspects of the work in ensuring that questions related to the accuracy or integrity of any part of the work are appropriately investigated and resolved. All procedures performed in this study involving human participants were in accordance with the ethical standards of the institutional research committee and with the Helsinki Declaration (as revised in 2013). Written informed consent was obtained from the patient for publication of this case report and accompanying images. A copy of the written consent is available for review by the editorial office of this journal.

Open Access Statement: This is an Open Access article distributed in accordance with the Creative Commons Attribution-NonCommercial-NoDerivs 4.0 International License (CC BY-NC-ND 4.0), which permits the noncommercial replication and distribution of the article with the strict proviso that no changes or edits are made and the original work is properly cited (including links to both the formal publication through the relevant DOI and the license). See: https://creativecommons.org/licenses/by-nc-nd/4.0/.

\section{References}

1. Demmy TL, James TA, Swanson SJ, et al. Troubleshooting video-assisted thoracic surgery lobectomy. Ann Thorac Surg 2005;79:1744-52; discussion 1753.

2. Mei J, Pu Q, Liao H, et al. A novel method for troubleshooting vascular injury during anatomic thoracoscopic pulmonary resection without conversion to thoracotomy. Surg Endosc 2013;27:530-7.

3. Liu L, Che G, Pu Q, et al. A new concept of endoscopic lung cancer resection: Single-direction thoracoscopic lobectomy. Surg Oncol 2010;19:e71-e77.

4. Ma L, Mei J, Liu C, et al. Precontrol of the pulmonary artery during thoracoscopic left upper lobectomy and systemic lymph node dissection. J Thorac Dis 2016;8:E317-E318.

5. Tam JK, Atasha A, Tan AK. Video-assisted thoracic surgery repair of subclavian artery injury following central venous catheterization: a new approach. Interact Cardiovasc Thorac Surg 2013;17:13-5.

Cite this article as: Zhu Y, Zheng Q, Liao H, Mei J, Kocher GJ, Shimizu K, Ma L. Successful thoracoscopic management of iatrogenic left subclavian arterial injury: a case report. J Thorac Dis 2022;14(1):194-198. doi: 10.21037/jtd-211922 